\title{
Egg white-derived peptides, QVPLW and LCAY, inhibit the activity of angiotensin-I converting enzyme in human umbilical vein endothelial cells by suppressing its recruitment into lipid rafts
}

Feng Jiang ${ }^{1}$, Ying Wang ${ }^{2}$, Chang Liu $^{1}$, Biying Zhang ${ }^{1}$, Erlei Wang ${ }^{1}$, Jingbo Liu $^{1} *$ \& Ting Zhang ${ }^{1, *}$

${ }^{1}$ Jilin Provincial Key Laboratory of Nutrition and Functional Food and College of Food Science and Engineering, Jilin University, Changchun 130062, People's Republic of China

${ }^{2}$ College of Food Engineering, Jilin Engineering Normal University, Changchun 130052, People's Republic of China

*Corresponding author:

Ting Zhang, E-mail address: tingzhang413@hotmail.com, tingzhang@jlu.edu.cn Jilin Provincial Key Laboratory of Nutrition and Functional Food and College of Food Science and Engineering, Jilin University, Changchun 130062, People's Republic of China

Jingbo Liu, E-mail address: 1jb168@sohu.com

Jilin Provincial Key Laboratory of Nutrition and Functional Food and College of Food Science and Engineering, Jilin University, Changchun 130062, People's Republic of China 
S1. Preparation, purification and identification of egg white peptides. The preparation, purification and identification of the hydrolysate were carried out according to the method of Ma et al. (Ma, S.; Liu, J.; Zhang, T.; Wang, Y.; Sun, Y.; Wei, Y.; Wang, H.; Lei, H.; Liu, B. Antioxidant Activity Differences and Peptide Sequence Analyses of Egg White Peptides between Simulated Gastrointestinal Digestion and Alkaline Protease Treatment and Peptide Sequence Analyses. Food Sci. (In Chinese) 2020, 41, 113-120.) Briefly, egg white powder (Jinyi, Jilin, China) was dispersed in distilled water to obtain $5 \%$ protein slurry $(\mathrm{w} / \mathrm{v})$ and hydrolyzed by alkaline proteinase Alcalase (Novozymes, Copenhagen, Denmark) at $50{ }^{\circ} \mathrm{C}$ for $3 \mathrm{~h}$. After that, the mixture was centrifuged $\left(12,000 \mathrm{~g}, 10 \mathrm{~min}, 4^{\circ} \mathrm{C}\right)$ and then the supernatant was separated with ultrafiltration membrane $(1 \mathrm{kDa})$. The protein fraction $(<1 \mathrm{kDa})$ was collected and freeze-dried for HPLC-MS/MS system consisted of an Easy nLC 1000 system and an Orbitrap Elite mass spectrometer (Thermo Scientific, MA, USA) equipped with a nanoESI source. The resulting MS/MS data were processed following an amino acid sequence analysis and a UniProt Gallus (Chicken) database search (http://www.uniprot.org/). Ultimately, eight peptides (QVPLW, LCAY, TYGC, VYEY, GPDVY, PGWV, CGDV, and GRVW) were identified (Table S1, Figure S1). 
Table S1. The parent protein and location of the identified peptides

\begin{tabular}{lllll}
\hline Number & Peptide & Protein & Uniprot ID & Location \\
\hline 1 & QVPLW & Vitellogenin-2 & P02845 & $1706-1710$ \\
2 & LCAY & Ovomucoid & P01005 & $67-70$ \\
3 & TYGC & Mucin-6 & F1NBL0 & $286-289$ \\
4 & VYEY & Albumin & P19121 & $359-362$ \\
5 & GPDVY & Ovostatin & P20740 & $653-657$ \\
6 & PGWV & Ovocleidin-17 & Q9PRS8 & $7-10$ \\
7 & CGDV & Mucin-6 & F1NBL0 & $992-995$ \\
8 & GRVW & Neuroligin 3 & D2X2H4 & $382-385$ \\
\hline
\end{tabular}


Table S2. ACE inhibition $\mathrm{IC}_{50}$ values of the selected 8 peptides

\begin{tabular}{llll}
\hline \multirow{2}{*}{ Number } & Peptide & Control & \multicolumn{1}{c}{$\mathrm{IC}_{50}(\mu \mathrm{M})$} \\
\hline 1 & QVPLW & $238.46 \pm 11.35$ & $45.43 \pm 6.15$ \\
2 & LCAY & $31.55 \pm 2.64$ & $34.63 \pm 1.59$ \\
3 & TYGC & ND & $454.51 \pm 44.08$ \\
4 & VYEY & $349.20 \pm 73.73$ & $358.64 \pm 105.09$ \\
5 & GPDVY & ND & ND \\
6 & PGWV & ND & ND \\
7 & CGDV & ND & ND \\
8 & GRVW & $401.56 \pm 73.54$ & ND \\
\hline
\end{tabular}

ND, not detected, indicating low ACE inhibitory activity. 

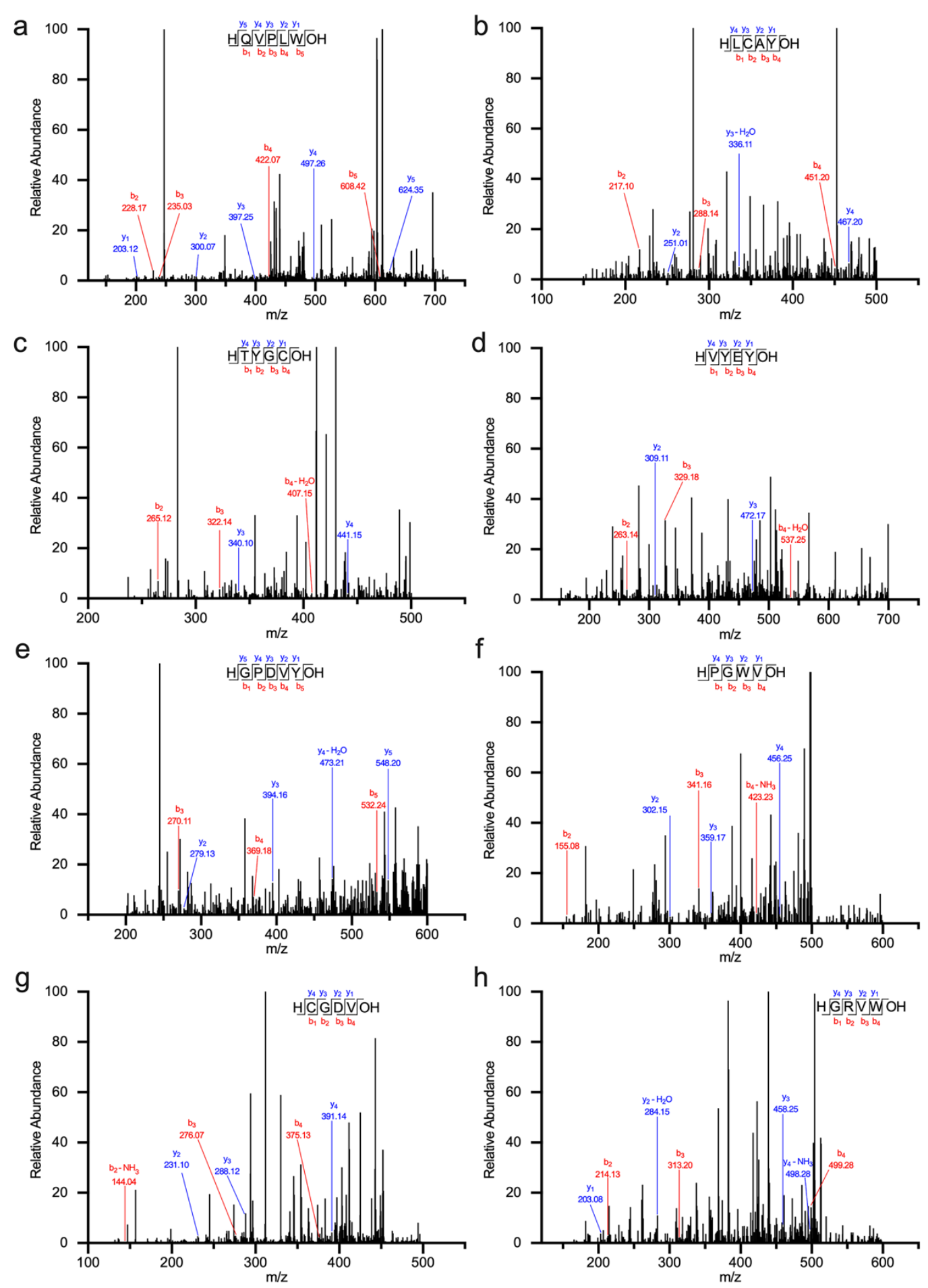

Figure S1. The MS/MS spectrum of the identified peptides. (a) QVPLW, (b) LCAY,

(c) TYGC, (d) VYEY, (e) GPDVY, (f) PGWV, (g) CGDV, and (h) GRVW 

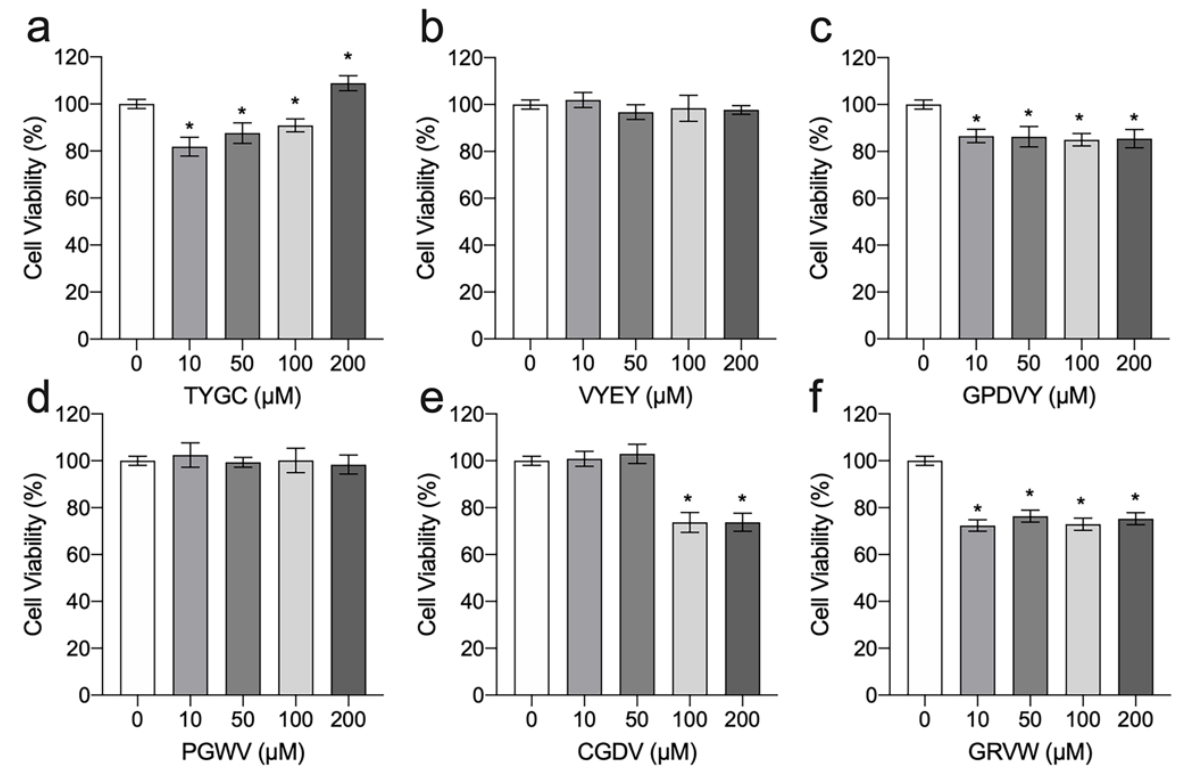

Figure S2. Effects of peptides (TYGC, VYEY, GPDVY, PGWV, CGDV and GRVW) on cell viability. ${ }^{*} \mathrm{p}<0.05$ vs Control- 0 . 

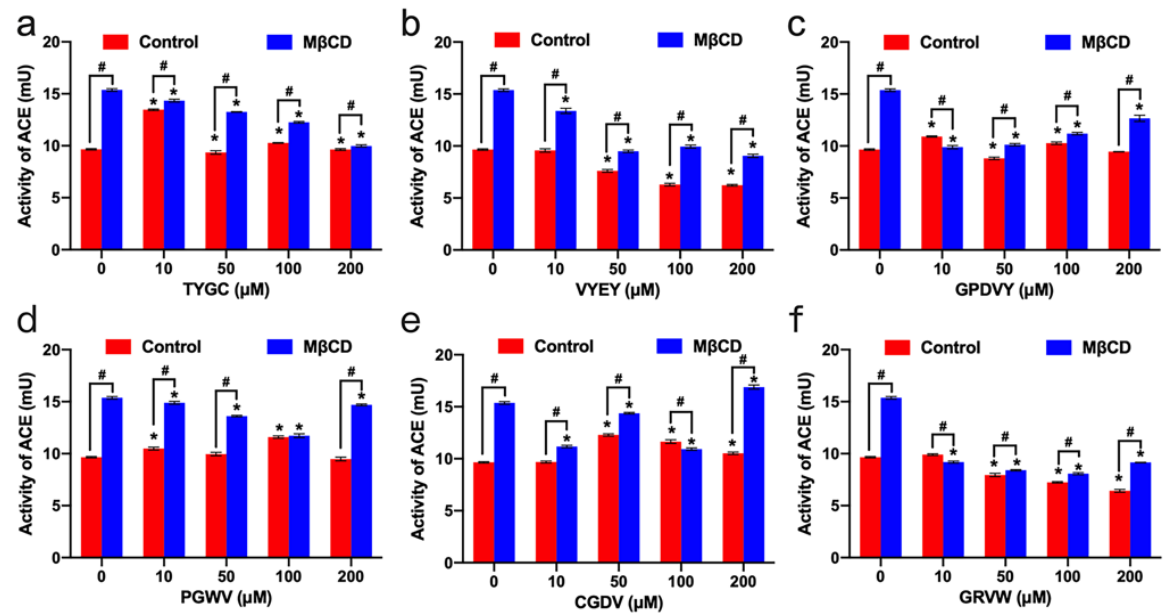

Figure S3. In situ ACE inhibitory activity of peptides (TYGC, VYEY, GPDVY, PGWV, CGDV and GRVW) in HUVECs. ${ }^{*} \mathrm{p}<0.05$ vs (Control-0 or M 3 CD-0). ${ }^{*} p<0.05$ compared between the Control group and $\mathrm{M} \beta \mathrm{CD}$ group. 